\title{
Probabilistic bisection converges almost as quickly as stochastic approximation
}

\author{
Peter I. Frazier, Shane G. Henderson, Rolf Waeber
}

\begin{abstract}
The probabilistic bisection algorithm (PBA) solves a class of stochastic root-finding problems in one dimension by successively updating a prior belief on the location of the root based on noisy responses to queries at chosen points. The responses indicate the direction of the root from the queried point, and are incorrect with a fixed probability. The fixed-probability assumption is problematic in applications, and so we extend the PBA to apply when this assumption is relaxed. The extension involves the use of a power-one test at each queried point. We explore the convergence behavior of the extended PBA, showing that it converges at a rate arbitrarily close to, but slower than, the canonical "square root" rate of stochastic approximation.
\end{abstract}

\section{Introduction.}

The probabilistic bisection algorithm (PBA) 7 is an algorithm for solving certain one-dimensional rootfinding problems where a query at a single point returns a stochastic response. The stochastic response is assumed to indicate the direction of the root, and is assumed correct with some fixed probability $p>1 / 2$. The PBA begins with a prior belief distribution on the location of the root, and repeatedly queries at the median of the belief distribution, updating the belief distribution using Bayes' rule. The sequence of medians of the belief distribution are now known to converge to the true root at an exponential rate 26.

The PBA itself, and algorithms based on the PBA, have been applied in contexts including target localization 23, scanning electron microscopy 22, topography estimation 4], edge detection 6], and value function approximation for optimal stopping problems 21. A modification of the PBA to the discrete setting was considered in [2, 3], as part of a larger body of work in computer science on unknownitem two-person question and answer games with lies [17. The PBA is known to be optimal in an average-case sense for an entropy loss function 8 .

The PBA is intuitive and the posterior belief distribution provides a great deal of insight on the location of the root. However, the assumption that the correctness probability $p$ does not depend on the location at which a query is performed limits its applicability. To see why, consider finding the root $x^{*}$ of a decreasing function $g:[0,1] \rightarrow \mathbb{R}$, where we observe $g(x)+\epsilon$ when querying $g$ at $x \in[0,1]$, where $\epsilon$ is a normal random variable with mean 0 and constant variance 1 that is independent of all previous queries and responses. The query indicates that $x^{*}>x$ if $g(x)+\epsilon>0$ and that $x^{*}<x$ if $g(x)+\epsilon<0$. If $g(x) \rightarrow 0$ as $x \rightarrow x^{*}$, then the probability that the query result is correct converges to $1 / 2$ as $x \rightarrow x^{*}$, violating the assumption of constant probability of correctness.

We modify the PBA to allow for non-constant probabilities of correctness. The modification performs repeated queries at a single point $x$ until the probability of correctness exceeds a user-selected threshold $p_{c}>1 / 2$. This can be achieved through the use of tests of power one. Once the test concludes at $x$, we update the belief distribution in the same manner as in the PBA, using success probability $p_{c}$. The resulting belief distribution is not a true posterior, and so the existing analysis of the PBA is not valid. Nevertheless, the belief distribution provides an intuitive representation of our belief about the location of the root, and can be leveraged to obtain confidence intervals on the location of the root 25 . These confidence intervals are valid irrespective of how many queries are performed, i.e., they are not asymptotic, and the resulting confidence interval widths shrink at an exponential rate in terms of the number of points at which the function is queried 25$]$.

In this paper, we show that when applying the modified PBA (henceforth referred to as the PBA for simplicity) on one-dimensional stochastic root-finding problems, the distance $\left|X_{n}-x^{*}\right|$ between the median $X_{n}$ of the belief distribution after running the test of power one at $n$ points and the true root 
$x^{*}$ converges at an exponential (in $n$ ) rate to 0 . (This result is not implied by the confidence interval result in 25.) This does not imply that we get an exponential rate of convergence in wall-clock time to the root, however, since the tests of power one have runlengths that increase without bound as $X_{n}$ approaches $x^{*}$. We further show that for any $\epsilon>0$, an estimator of the root based on the first $n$ tests that collectively require $T_{n}$ total queries (which we take as a reasonable surrogate for wall-clock time) has an error that is at most of stochastic order $T_{n}^{-1 / 2+\epsilon}$.

By way of contrast, the standard method for stochastic root-finding problems is stochastic approximation [19, 12, 16]. Under mild regularity conditions, stochastic approximation applied in our setting would have an error of stochastic order $c^{-1 / 2}$ based on a wall-clock running time of $c$. The $\epsilon$ difference in the exponent is striking, particularly since $\epsilon>0$ can be arbitrarily small, and explains the title of this paper. Our analysis does not provide a tight bound on the true stochastic order of the error in PBA, but we conjecture that it is $T_{n}^{-1 / 2} \ln \left(T_{n}\right)$, which is slightly slower than stochastic approximation.

Even though PBA appears to have a slower asymptotic convergence rate than stochastic approximation, we still believe the algorithm has value for several reasons. First, the belief distribution provides insight on the location of the root. Second, this asymptotic result does not shed light on how the algorithms compare over wall-clock running times that are representative in practice. Third, stochastic approximation requires the selection of a large number of tuning parameters, notably the "gain sequence," with poor selections leading to reductions in the rate of convergence; see, e.g., 1, p. 253]. Fourth, the PBA appears to be readily adapted for use in parallel computing environments [15], while the situation is more complicated for stochastic approximation [10, 11].

We view the key contributions of this paper to be the extension of the PBA to a broad class of root-finding problems, and the analysis of the asymptotic error, both as a function of the number of power-one tests completed, and as a function of $T_{n}$, the surrogate for wall-clock time. The convergence analysis herein is completely different from that in 26] for the original PBA due to necessity, and is a considerable outgrowth of the original source for these ideas in 25] in which the exponential convergence rate was conjectured but unproven. It is also completely different from the analysis in [2] where it is assumed that the root lies amongst a finite set of alternatives.

The question of whether the PBA can be successfully applied in root-finding problems of dimension greater than one remains open. We do not consider that question here.

The remainder of this paper is organized as follows. The PBA is reviewed in $\$ 2$ and in $\$ 3$ we provide our extension of the PBA that uses tests of power one to advance at each step. We establish the exponential (in $n$ ) rate of convergence of the sequence of medians in 4 , and consider wall-clock time behavior in 8 . Proofs that are omitted to this point are collected in 46

\section{Probabilistic Bisection.}

Assumption 1. Let $g:[0,1] \rightarrow \mathbb{R}$ and suppose that there exists $x^{*} \in[0,1]$ such that $g(x)>0$ for $x<x^{*}$ and $g(x)<0$ for $x>x^{*}$.

Our goal is to estimate $x^{*}$, but we make no assumption about the value of $g\left(x^{*}\right)$.

In this section we assume that we can query the value of $g$ at any point $x \in(0,1)$, obtaining a signal $Z(x) \in\{-1,1\}$, independently of all previous queries. The signal indicates the likely direction of the root $x^{*}$ from $x$. If $x^{*}>x$, then $Z(x)=1$ with probability $p$ and $Z(x)=-1$ with probability $q=1-p$, for some fixed $p \in(1 / 2,1]$. Similarly, if $x^{*}<x$, then $Z(x)=-1$ with probability $p$, and $Z(x)=1$ with probability $q$. If $x=x^{*}$ then we arbitrarily assume $Z\left(x^{*}\right)=1$ with probability $p$.

The PBA begins with a prior density $f_{0}$ on $[0,1]$ that is positive everywhere. Let $F_{0}$ denote the corresponding cumulative distribution function. Then, for $n=0,1,2, \ldots$, it iterates as follows.

1. Determine the next measurement point $X_{n}$ as the median of $f_{n}, X_{n}=F_{n}^{-1}(1 / 2)$.

2. Query at the point $X_{n}$ to obtain $Z_{n}=Z_{n}\left(X_{n}\right)$.

3. Update the density $f_{n}$ :

$$
\begin{aligned}
& \text { if } Z_{n}\left(X_{n}\right)=+1, \text { then } f_{n+1}(y)= \begin{cases}2 p f_{n}(y), & \text { if } y \geq X_{n}, \\
2 q f_{n}(y), & \text { if } y<X_{n},\end{cases} \\
& \text { if } Z_{n}\left(X_{n}\right)=-1, \text { then } f_{n+1}(y)= \begin{cases}2 q f_{n}(y), & \text { if } y \geq X_{n}, \\
2 p f_{n}(y), & \text { if } y<X_{n}\end{cases}
\end{aligned}
$$

4. $n \leftarrow n+1$ 
The updating of the density is quite natural, scaling up the mass in the perceived direction of the root, and scaling down the mass in the other direction. In fact, this update gives the Bayesian posterior distribution of the location of the root under appropriate assumptions; see 26].

The assumption that the signal has constant probability of being correct, irrespective of how close $x$ is to $x^{*}$, is problematic in root-finding problems, and we now weaken that assumption.

\section{The Algorithm.}

Now suppose that when we query $g$ at a point $x \in[0,1]$, we obtain $Y(x)=g(x)+\epsilon(x)$, where $\epsilon(x)$ is a random variable, independent of any previous function evaluations, that represents the observation noise. Repeated observations at a fixed point $x$ yield i.i.d. observations $\left(Y_{i}(x)=g(x)+\epsilon_{i}(x): i=1,2, \ldots, m\right)$.

Let $\tilde{Z}(x)=2 \mathbb{I}(Y(x) \geq 0)-1$, so that $\tilde{Z}(x)=1$ if $Y(x) \geq 0$ (so that we believe $x^{*}$ to be to the right of $x)$ and $\tilde{Z}(x)=-1$ if $Y(x)<0$. Also, define

$$
\tilde{p}(x)= \begin{cases}P(\tilde{Z}(x)=1) & \text { if } x \leq x^{*}, \\ P(\tilde{Z}(x)=-1) & \text { if } x>x^{*},\end{cases}
$$

so that $\tilde{p}(x)$ gives the probability that $\tilde{Z}(x)$ correctly indicates the direction of the root $x^{*}$ from $x$.

Assumption 2. For any $x \neq x^{*}, \tilde{p}(x)>1 / 2$.

Assumption 2 can be assured through conditions on the distribution of the noise random variables $\epsilon(x)$. For example, the assumption holds if $\epsilon(x)$ has, for all $x$, median 0 and a strictly positive density component in a neighborhood of 0 , as would be the case with unbiased normally distributed estimators of $g(x)$. To see why we might need the positive density condition or a similar regularity condition, suppose that $\epsilon(x)$ has, for all $x$, a density that is positive only on $(-\infty,-1) \cup(1, \infty)$ with (non-unique) median 0 . In this case, if $|g(x)|<1$ then $p(x)=1 / 2$.

We would like to use $\tilde{p}(x)$ in place of $p$ in the update step of the PBA (12), but $\tilde{p}(x)$ is unknown. Furthermore, under reasonable assumptions about the errors $\epsilon(x)$, when $g$ is continuous at $x^{*}$ one expects that $\tilde{p}(x) \rightarrow 1 / 2$ as $x \rightarrow x^{*}$, so that the density updates become less and less effective as $x \rightarrow x^{*}$.

To overcome this difficulty, we replace the signal $\tilde{Z}(x)$ with a new signal, $Z(x)$, which has probability $p(x)$ of being correct. Moreover, $p(x) \geq p_{c}>1 / 2$ for all $x \in[0,1] \backslash\left\{x^{*}\right\}$ for some $p_{c}$ chosen by the user, so that $p(x)$ is bounded away from $1 / 2$. Even though $p(x)$ is unknown, $p_{c} i s$ known, and we can then update the density using (1)-(2) using some $p \in\left(1 / 2, p_{c}\right)$. This update does not represent a true Bayesian update to a posterior distribution, but can be viewed as a natural extension of the PBA for problems with nonconstant and unknown $p(\cdot)$.

To obtain $Z(x)$ for $x \neq x^{*}$, we repeatedly evaluate the function $g$ at $x$, observing a sequence of signs $\left(\tilde{Z}_{i}(x)\right)_{i}$. If $x<x^{*}$ then $E\left[\tilde{Z}_{i}(x)\right]=2 \tilde{p}(x)-1>0$ and if $x>x^{*}$ then $E\left[\tilde{Z}_{i}(x)\right]=1-2 \tilde{p}(x)<0$. Our goal at a particular $x \neq x^{*}$ is then to evaluate the sign of the drift of the simple random walk $S(x)=\left(S_{m}(x): m \geq 0\right)$ with $S_{0}(x)=0$ and $S_{m}(x)=\sum_{i=1}^{m} \tilde{Z}_{i}(x)$ for $m \geq 1$. Sequential tests of power one provide a powerful tool to decide whether the drift $\theta$ of a random walk satisfies the hypothesis $\theta<0$ versus $\theta>0$.

In a slight abuse of notation, let $S(\theta)$ denote a generic random walk with increments in $\{-1,1\}$ and drift $\theta$. A test of power one can be defined through a positive sequence $\left(k_{m}\right)_{m}$ and a stopping time $\mathcal{N}(\theta)=\inf \left\{m \in \mathbb{N}:\left|S_{m}(\theta)\right| \geq k_{m}\right\}$ [25]. The test decides that $\theta>0$ if $S_{\mathcal{N}(\theta)}(\theta) \geq k_{\mathcal{N}(\theta)}$, that $\theta<0$ if $S_{\mathcal{N}(\theta)}(\theta) \leq-k_{\mathcal{N}(\theta)}$ and does not make a decision if $\mathcal{N}(\theta)=\infty$. Furthermore, for a chosen confidence parameter $\gamma \in(0,1)$ such a test satisfies $P(\mathcal{N}(\theta)<\infty) \leq \gamma$ if $\theta=0$ and $P(\mathcal{N}(\theta)<\infty)=1$ if $\theta \neq 0$. In Section 6 we show that one may take

$$
k_{m}=(2 m(\ln (m+1)-\ln \gamma))^{1 / 2}
$$

This is not an optimal test. Indeed, as shown more generally in [20, 13, the expected value of the stopping time of our test when the true drift is $\theta \neq 0$ is $O\left(\theta^{-2} \ln \left(|\theta|^{-1}\right)\right)$ as $\theta \rightarrow 0$, whereas [5] establishes that an optimal test should have expected stopping time of the order $O\left(\theta^{-2}|\ln | \ln |\theta|||\right)$. Unfortunately, optimal tests are difficult to implement in practice because they rely on constants that are difficult to compute. The power-one test we choose is sufficient for our purposes.

One might expect that a more powerful signal could be obtained by working directly with the observations $Y(x)=g(x)+\epsilon(x)$, rather than with the sign of the observations. One could envisage using other power-one tests built on repeated iid observations of $Y(x)$, but we expect [5, 13, 20] that while this 
might accelerate the method, it would not change the order of the rate of convergence. Accordingly, we work with the construction above.

Assume now that the PBA measures at some point $X_{n} \neq x^{*}$ at the $(n+1)$ st iteration. We then observe a random walk with $m$ th term $S_{n, m}=S_{n, m}\left(X_{n}\right)=\sum_{i=1}^{m} Z_{n, i}\left(X_{n}\right)$ until the test of power one terminates. Denote the stopping time of the power one test by $N_{n}$, which is almost surely finite since $X_{n} \neq x^{*}$, and define the new signal

$$
Z_{n}\left(X_{n}\right)= \begin{cases}+1, & \text { if } S_{n, N_{n}}>0 \\ -1, & \text { if } S_{n, N_{n}}<0\end{cases}
$$

Recall that $S(0)$ is a zero-drift simple random walk. On the event $X_{n}>x^{*}$,

$$
\begin{aligned}
P\left(Z_{n}\left(X_{n}\right)=+1 \mid X_{n}\right) & =P\left(S_{n, N_{n}}>0, N_{n}<\infty \mid X_{n}\right) \\
& \leq P\left(S_{\mathcal{N}(0)}(0)>0, \mathcal{N}(0)<\infty\right) \\
& \leq \gamma / 2
\end{aligned}
$$

where the first inequality follows by a sample path argument and the second inequality by the property that $P(\mathcal{N}(0)<\infty) \leq \gamma$. An analogous argument shows that on the event $X_{n}<x^{*}$,

$$
P\left(Z_{n}\left(X_{n}\right)=-1 \mid X_{n}\right) \leq \gamma / 2 .
$$

So, for $x \in[0,1] \backslash\left\{x^{*}\right\}$, we have $p(x) \geq 1-\gamma / 2$, where

$$
p(x)= \begin{cases}P\left(Z_{n}(x)=+1\right), & \text { if } x<x^{*}, \\ P\left(Z_{n}(x)=-1\right), & \text { if } x>x^{*}\end{cases}
$$

Defining $p_{c}=1-\gamma / 2$ it follows that $p(x) \geq p_{c}$ for all $x \neq x^{*}$, where $p_{c} \in(1 / 2,1)$ is a chosen constant (since one can choose $\gamma \in(0,1)$ in the construction of the test of power one).

It remains to discuss the case where $X_{n}=x^{*}$. In this case the test of power one might not terminate and the search algorithm might stall. From a theoretical point of view this is not convenient because the sequence $\left(X_{n}\right)_{n}$ is then not well-defined for all $n \geq 0$. In practice, the stalling of the algorithm can be desirable since in this case the point $x^{*}$ is successfully located. (Of course, stalling at $x^{*}$ cannot be detected, because it is impossible to test in finite time whether the stopping time is finite or not.) The event that $X_{n}=x^{*}$ for some finite $n$ seems very unlikely in practice. Consider, for example, the case where $x^{*}$ is a realization of a random variable with a positive density on [0,1]: Then the probability that $X_{n}=x^{*}$ for any $n \in \mathbb{N}$ is zero, since $X_{n}$ can only assume values on a set of cardinality $2^{n}$. We therefore adopt Assumption 3 below. This can be assured, e.g., by artificially inflating the interval $[0,1]$ to $[0,1+\varepsilon]$ for some $\varepsilon$ that is uniformly distributed on $[0, \zeta]$ for any $\zeta>0$, taking $\tilde{p}(x)=1$ for $x>1$, and finally rescaling so that the interval $[0,1+\varepsilon]$ is mapped back to $[0,1]$.

Assumption 3. $X_{n} \neq x^{*}$ for all $n=0,1,2, \ldots$ almost surely.

To summarize the algorithm then, we begin with a uniform prior on the location of the root, and then repeatedly perform a power-one test at the median, updating the prior using some $p \in\left(1 / 2, p_{c}\right)$, where $p_{c}$ is the lower bound on $p(\cdot)$ used in the power-one test.

Using the PBA with signals $\left(Z_{n}\left(X_{n}\right)\right)_{n}$ instead of $\left(\tilde{Z}_{n}\left(X_{n}\right)\right)_{n}$ introduces two time scales, namely, the macro time $n$ corresponding to the number of different measurement points $\left(X_{n}\right)_{n}$ and the wall-clock time $T$ counting the total number of function evaluations.

Intuitively, the closer the current measurement point $X_{n}$ is to $x^{*}$, the closer $\tilde{p}\left(X_{n}\right)$ is to $1 / 2$, and the longer the test of power one requires to terminate. In fact, the expected hitting time is larger than $O\left((\tilde{p}(x)-1 / 2)^{-2}\right)$ 13, 20. So, if $\tilde{p}(x) \rightarrow 1 / 2$ as $x \rightarrow x^{*}$, as would typically hold if $g$ is continuous at $x^{*}$, then the number of function evaluations between two macro iterations is likely to become very large, which will lead to a discrepancy in the convergence behaviors in the two time scales.

\section{Geometric Convergence.}

Recall that $X_{n}$ gives the $n$th measurement point, with $X_{n}$ being the median of the distribution $\mu_{n}$ associated with the $n$th density $f_{n}, n \geq 0$. In this section we establish geometric a.s. convergence of $X_{n} \rightarrow x^{*}$. This geometric convergence arises in the macro time scale, i.e., the quantity $n$ gives the number of power-one tests that are applied. 
Theorem 1. Suppose Assumptions 1-3 hold and that we begin with a uniform prior. Then there exists $r>0$ such that $e^{r n}\left(X_{n}-x^{*}\right) \rightarrow 0$ as $n \rightarrow \infty$ a.s.

Proof. Proof of Theorem 1. We assume that $x^{*} \in(0,1)$. A very similar, but simpler, argument that we omit can be employed to prove the result if $x^{*} \in\{0,1\}$. Fix $a \in\left(0, x^{*}\right)$ and define $A=\left[0, x^{*}-a\right], B=$ $\left(x^{*}-a, x^{*}\right], C=\left(x^{*}, 1\right]$. Define $T(a)=\inf \left\{n^{\prime} \geq 0: X_{n} \in B \cup C \forall n \geq n^{\prime}\right\}$ to be the time required until the sequence of medians never re-enters $A$. Let $\stackrel{\text { st }}{\leq}$ denote stochastic ordering, so that $X \stackrel{\text { st }}{\leq} Y$ iff $P(X>x) \leq P(Y>x)$ for all $x$. We will show that

$$
T(a) \stackrel{\text { st }}{\leq} \tau(a)+R
$$

where $\tau(a)$ and $R$ are independent random variables with appropriately light tails, and the nonnegative random variable $R$ does not depend on $a$. This implies that for some $r>0, e^{r n}\left|X_{n}-x^{*}\right| I\left(X_{n} \leq x^{*}\right) \rightarrow 0$ as $n \rightarrow \infty$ a.s., since for arbitrary $\epsilon>0$,

$$
\begin{aligned}
P\left(e^{r n}\left|X_{n}-x^{*}\right| \mathbb{I}\left(X_{n} \leq x^{*}\right)>\epsilon\right) & =P\left(x^{*}-X_{n}>\epsilon e^{-r n}\right) \\
& \leq P\left(T\left(\epsilon e^{-r n}\right)>n\right) \\
& \leq P\left(\tau\left(\epsilon e^{-r n}\right)+R>n\right) \\
& \leq P\left(\tau\left(\epsilon e^{-r n}\right)>n / 2\right)+P(R>n / 2) .
\end{aligned}
$$

Lemma 9 shows that $R$ has an exponentially decaying tail and thus $P(R>n / 2)$ is finitely summable in $n$. Lemma 3 establishes that the first term in (7) is finitely summable in $n$. The Borel-Cantelli lemma allows us to conclude that $e^{r n}\left|X_{n}-x^{*}\right| \mathbb{I}\left(X_{n} \leq x^{*}\right) \rightarrow 0$ as $n \rightarrow \infty$ a.s. The same overall argument can be used to give the symmetric result that $e^{r n}\left|X_{n}-x^{*}\right| \mathbb{I}\left(X_{n}>x^{*}\right) \rightarrow 0$ as $n \rightarrow \infty$ a.s.

The remainder of this section is dedicated to the supporting results needed to establish Theorem 1 The constant $a$ and sets $A, B$ and $C$ defined in the proof of Theorem 1 are fixed and employed throughout the remainder of this section. In the argument, we decompose the time till we never return to $A$ by a sum of two terms. The first term, $\tau(a)$, gives the time required to get sufficient conditional mass in the set $B$, i.e., sufficient mass in $B$ relative to the mass in $A \cup B$. From this point, we show that there will be a finite number of excursions during which the conditional mass in $A$ climbs back up above $1 / 2$, before the conditional mass in $A$ never climbs that high again, and hence the sequence of medians never returns to $A$.

Let $(\Omega, \mathcal{F}, P)$ be an underlying probability space with a filtration $\mathcal{G}=\left(\mathcal{G}_{n}: n \geq 0\right)$. Let $\mu_{n}$ denote the random measure associated with the $n$th density $f_{n}, n \geq 0$. So for each fixed $n \geq 0$ and $\omega \in \Omega$, $\mu_{n}(\cdot, \omega)$ is a probability measure on the Borel sets of $[0,1]$, and for each Borel set $D \subset[0,1], \mu_{n}(D, \cdot)$ is a $\mathcal{G}_{n}$-measurable random variable. The median $X_{n}$ of $\mu_{n}$ is $\mathcal{G}_{n}$ measurable, and is uniquely defined because $f_{n}$ is strictly positive, for all $n \geq 0$. The response $Z_{n}\left(X_{n}\right)$ is $\mathcal{G}_{n+1}$ measurable for each $n \geq 0$.

We first show that the sequence of medians spends linear time on the left side of the root $x^{*}$ with very high probability. Fix $\delta \in(0,1 / 2)$ and, for $n \geq 1$, let

$$
N(n)=\sum_{k=0}^{n-1} \mathbb{I}\left(\mu_{k}\left(\left[0, x^{*}\right]\right) \geq 1 / 2+\delta\right)
$$

be the number of instances from time 0 to time $n-1$ when the mass of $A \cup B$ is at least $1 / 2+\delta$. (It is important that the upper index $n-1$ is not $n$ when we define certain supermartingales below.) Define $N(0)=0$.

Lemma 1. We can choose $\delta>0$ in the definition of $N(\cdot)$ above such that there exist constants $\alpha>$ $0, r_{1}>0$ depending on $x^{*}$ and $\delta$ but not on a such that $P(N(n) \leq \alpha n) \leq \varphi\left(\mu_{0}(A \cup B)\right) e^{-r_{1} n}$. The function $\varphi(\cdot)$ is given in (25), does not depend on a, and in particular is bounded above when $\mu_{0}(A \cup B)$ is bounded away from 0 .

Define $\nu_{n}$ to be the (random) measure corresponding to the conditional posterior distribution, conditional on lying to the left of $x^{*}$, so that for any measurable $D, \nu_{n}(D)=\mu_{n}\left(D \cap\left[0, x^{*}\right]\right) / \mu_{n}\left(\left[0, x^{*}\right]\right)$. Next, fix $\Delta \in(0,1 / 2)$ and define the stopping time $\tau=\tau(a)$ to be the first time that the conditional mass in $B$ lies above $1-\Delta$ and the median $X_{n}$ lies to the left of $x^{*}$, i.e.,

$$
\tau=\inf \left\{n \geq 0: \nu_{n}(B)>1-\Delta, X_{n}<x^{*}\right\} .
$$

For real numbers $b, c$, let $b \wedge c=\min \{b, c\}$. 
Lemma 2. For $n \geq 0$, define $M_{n}=e^{r N(n \wedge \tau)} / \nu_{n \wedge \tau}(B)$. Suppose that $p(\cdot) \geq p_{c}>p>1 / 2$. Then there exists $r=r_{2}>0$ such that $\left(M_{n}: n \geq 0\right)$ is a supermartingale with respect to the filtration $\mathcal{G}$. The constant $r_{2}$ depends only on $\Delta, \delta, p_{c}$ and $p$.

Lemma 3. Under the uniform prior, $E e^{r_{2} N(\tau)} \leq x^{*} / a$ and the first term in (7) is summable.

Proof. Proof. From the supermartingale property and the uniform prior, we have that

$$
E e^{r_{2} N(n \wedge \tau)} \leq E \frac{e^{r_{2} N(n \wedge \tau)}}{\nu_{n \wedge \tau}(B)}=E M_{n} \leq E M_{0}=\frac{1}{\nu_{0}(B)}=\frac{x^{*}}{a}
$$

But $N(\cdot)$ is nondecreasing, so $e^{r_{2} N(n \wedge \tau)} \nearrow e^{r_{2} N(\tau)}$ as $n \rightarrow \infty$. Monotone convergence then yields

$$
E e^{r_{2} N(\tau)}=\lim _{n \rightarrow \infty} E e^{r_{2} N(n \wedge \tau)} \leq x^{*} / a
$$

For the particular choice $a=\epsilon e^{-r n}$, we obtain $E e^{r_{2} N\left(\tau\left(\epsilon e^{-r n}\right)\right)} \leq e^{r n} x^{*} / \epsilon$, and hence

$$
\begin{aligned}
P\left(\tau\left(\epsilon e^{-r n}\right)>n / 2\right) & \leq P\left(N\left(\tau\left(\epsilon e^{-r n}\right)\right) \geq N(n / 2)\right) \\
& \leq P\left(N\left(\tau\left(\epsilon e^{-r n}\right)\right) \geq N(n / 2), N(n / 2)>\alpha n / 2\right)+P(N(n / 2) \leq \alpha n / 2) \\
& \leq P\left(N\left(\tau\left(\epsilon e^{-r n}\right)\right)>\alpha n / 2\right)+\beta e^{-r_{1} n / 2} \\
& \leq e^{-r_{2} \alpha n / 2} E e^{r_{2} N\left(\tau\left(\epsilon e^{-r n}\right)\right)}+\beta e^{-r_{1} n / 2} \\
& \leq e^{-r_{2} \alpha n / 2} \frac{x^{*}}{\epsilon} e^{r n}+\beta e^{-r_{1} n / 2} .
\end{aligned}
$$

Here we used the increasing nature of $N(\cdot)$ in (9) and a Markov bound in (10). Taking $r<\alpha r_{2} / 2$ gives the desired summability.

We now need to show the summability of the second term in (7). Define the stopping time $\eta$ to be the first time that the conditional mass in $A$ reaches $1 / 2$ or more, i.e., $\eta=\inf \left\{n \geq 0: \nu_{n}(A) \geq 1 / 2\right\}$. Implicit in this definition is the fact that $\eta=\infty$ if $\nu_{n}(A)<1 / 2$ for all $n$. (The specific value $1 / 2$ is chosen so that for $n<\eta$ we have $\mu_{n}(A) \leq \nu_{n}(A)<1 / 2$, so that $X_{n} \in B \cup C$ for $n<\eta$.)

Lemma 4. For $n \geq 0$, define $L_{n}=e^{r N(n \wedge \eta)} \nu_{n \wedge \eta}(A)$. There exists $r=r_{3}>0$ such that $\left(L_{n}: n \geq 0\right)$ is a supermartingale with respect to the filtration $\mathcal{G}$. The constant $r_{3}$ depends only on $p_{c}, p$ and $\delta$.

Define $U_{0}=\tau=\inf \left\{n \geq 0: \nu_{n}(B) \geq 1-\Delta, X_{n}<x^{*}\right\}$. Lemma 3 establishes that $U_{0}$ is finite a.s. Now, for $n \geq 1$, recursively define

$$
\begin{aligned}
V_{n} & =\inf \left\{k>U_{n-1}: \nu_{k}(A) \geq 1 / 2\right\}, \text { and } \\
U_{n} & =\inf \left\{k>V_{n}: \nu_{k}(B) \geq 1-\Delta\right\} .
\end{aligned}
$$

Here $V_{n}$ is the first time after time $U_{n-1}$ that the conditional mass in $A$ becomes at least $1 / 2$, and $U_{n}$ is the first time after $V_{n}$ that the conditional mass in $B$ is once again at least $1-\Delta$. Implicit in the definition of $U_{n}$ and $V_{n}$ for $n \geq 1$ is that they take the value $\infty$ if the stated event does not occur. Let $\Gamma$ be the number of "cycles," i.e., $\Gamma=\sum_{n=1}^{\infty} \mathbb{I}\left(V_{n}<\infty\right)$.

We can now write

$$
T(a)=U_{0}+\sum_{j=1}^{\Gamma}\left[\left(V_{j}-U_{j-1}\right)+\left(U_{j}-V_{j}\right)\right] .
$$

In this expression, $V_{j}-U_{j-1}$ represents the $j$ th time required to increase the conditional mass in $A$ from below $\Delta$ to $1 / 2$ or more, while $U_{j}-V_{j}$ is the subsequent time required to ensure that the conditional mass in $B$ is at least $\Delta$. We take the empty sum (when $\Gamma=0$ ) to equal 0 .

Consider $U_{j}-V_{j}$, conditional on $\Gamma \geq j$, i.e., conditional on both $U_{j}$ and $V_{j}$ being finite. This quantity represents the number of steps required to return the conditional mass in $B$ to at least $1-\Delta$, starting from a point where the conditional mass in $A$ is at least $1 / 2$.

Lemma 5. Conditional on $\Gamma \geq n, U_{j}-V_{j} \stackrel{s t}{\leq} \xi_{j}$ for $1 \leq j \leq n$, where $\xi_{1}, \xi_{2}, \ldots$ are iid random variables with exponential tails, the decay rate of which does not depend on a.

Our next result establishes that $\eta$ has a geometrically decaying tail. 
Lemma 6. For $n \geq 0$,

$$
P(\eta=n) \leq 2 \nu_{0}(A) e^{-r_{3} \alpha n}+\beta e^{-r_{1} n} .
$$

Here the constant $\beta$ depends on $\mu_{0}\left(\left[0, x^{*}\right]\right)$.

Proof. Proof. In this result we do not assume a uniform prior, because we will apply the result with varying initial conditions. First note that $P(\eta=0)=\mathbb{I}\left(\nu_{0}(A) \geq 1 / 2\right) \leq 2 \nu_{0}(A)$, so the result is immediate. For $n \geq 1$,

$$
\begin{aligned}
P(\eta=n)= & P\left(\nu_{n}(A) \geq 1 / 2, \eta \geq n\right) \\
= & P\left(\nu_{n \wedge \eta}(A) \geq 1 / 2, \eta \geq n\right) \\
\leq & P\left(e^{r_{3} N(n \wedge \eta)} \nu_{n \wedge \eta}(A) \geq \frac{1}{2} e^{r_{3} N(n \wedge \eta)}, N(n \wedge \eta) \geq \alpha n, \eta \geq n\right) \\
& \quad+P(N(n \wedge \eta)<\alpha n, \eta \geq n) \\
\leq & P\left(L_{n} \geq \frac{1}{2} e^{r_{3} \alpha n}\right)+P(N(n)<\alpha n) \\
\leq & 2 E\left(L_{n}\right) e^{-r_{3} \alpha n}+\beta e^{-r_{1} n} \\
\leq & 2 E\left(L_{0}\right) e^{-r_{3} \alpha n}+\beta e^{-r_{1} n} \\
= & 2 \nu_{0}(A) e^{-r_{3} \alpha n}+\beta e^{-r_{1} n}
\end{aligned}
$$

We used Markov's inequality in (11).

Next consider $V_{j+1}-U_{j}$ on the event $V_{j+1}<\infty$, for $j \geq 0$. This quantity gives the number of steps required for the conditional mass in $A$ to re-enter $[1 / 2,1]$ after going below $\Delta$.

Lemma 7. Suppose $\nu_{0}(B) \geq 1-\Delta$ and $\mu_{0}(A \cup B)$ is bounded away from 0 . Then there exists $r_{4}>0$ such that

$$
E\left[e^{r_{4}\left(V_{j+1}-U_{j}\right)} ; V_{j+1}<\infty\right]
$$

is bounded for all $j \geq 0$.

Proof. Proof. Suppose that we re-initiate the process at time 0 under the posterior at time $U_{j}$. From Lemma 6.

$$
\begin{aligned}
E\left[e^{r\left(V_{j+1}-U_{j}\right)} ; V_{j+1}<\infty\right] & =\sum_{n=0}^{\infty} e^{r n} P(\eta=n) \\
& \leq \sum_{n=0}^{\infty} e^{r n}\left[2 \nu_{0}(A) e^{-r_{3} \alpha n}+\varphi\left(\mu_{0}(A \cup B)\right) e^{-r_{1} n}\right] .
\end{aligned}
$$

But $\nu_{0}(A) \leq \Delta$ and $\varphi\left(\mu_{0}(A \cup B)\right)$ is bounded, since $\mu_{0}(A \cup B)$ is bounded away from 0 by assumption. Choosing $r=r_{4}<\min \left\{r_{1}, r_{3}\right\}$ gives the result.

The assumptions of Lemma 7 are satisfied at each of the times $U_{0}, U_{1}, \ldots$ that are finite. This follows by definition of $U_{0}$, and for $j \geq 1$, it follows since the conditional mass in $B$ can only strictly increase when the median is to the left of $x^{*}$, so that the mass in $A \cup B$ immediately before time $U_{j}$ is at least $1 / 2$ and therefore at least $q$ at time $U_{j}$.

Our next result establishes that when $\nu_{0}(A)<1 / 2$, the probability that the sequence of medians ever returns to $A$ is bounded away from 1 , so that the number of cycles $\Gamma$ is stochastically bounded by a geometric random variable that does not depend on $a$.

Lemma 8. If $\mu_{0}(A \cup B) \geq 1 / 2$ and $\nu_{0}(B)>1-\Delta$ then $P(\eta<\infty) \leq \rho$ for some constant $\rho<1$.

Proof. Proof. From Lemma 6 ]

$$
P(\eta<\infty)=\sum_{n=0}^{\infty} P(\eta=n) \leq P(\eta<K)+\sum_{n=K}^{\infty}\left[2 \nu_{0}(A) e^{-r_{3} \alpha n}+\beta\left(\mu_{0}(A \cup B)\right) e^{-r_{1} n}\right]
$$

for any fixed $K>0$. Now, $\nu_{0}(A) \leq \Delta$ and $\beta\left(\mu_{0}(A \cup B)\right)$ is bounded because of the assumption that $\mu_{0}(A \cup B) \geq 1 / 2$. Choose $K$ so that the infinite sum is at most $1 / 2$. Then choose $\Delta$ small enough that $P(\eta<K)=0$. (This is possible since $\eta=\inf \left\{n \geq 0: \nu_{n}(A) \geq 1 / 2\right\}$, and $\nu_{n}(A)$ increases by at most a factor $p / q$ at each step, and will therefore hold if $\Delta(p / q)^{K}<1 / 2$. 
Lemma 9. Under the assumptions of Theorem 1, the second term in (6) has a finite moment generating function in a neighborhood of 0 .

Proof. Proof. Recall that

$$
T(a)=U_{0}+\sum_{j=1}^{\Gamma}\left[\left(V_{j}-U_{j-1}\right)+\left(U_{j}-V_{j}\right)\right] .
$$

Lemma 8 establishes that $\Gamma$ is stochastically dominated by a geometric random variable, $\bar{\Gamma}$ say, such that $P(\bar{\Gamma}=k)=(1-\rho) \rho^{k}$ for $k \geq 0$. Also, Lemmas [5 and 7 together establish that the summands are stochastically bounded by an iid sequence with exponentially decaying tails where the decay rate does not depend on $a$. Such a geometric sum has moments of all orders which do not depend on $a$. The geometric sum plays the role of the random variable $R$ in (6).

\section{Near-Canonical Convergence.}

Theorem 1 establishes that the sequence $\left(X_{n}: n \geq 0\right)$ converges to the true root $x^{*}$ at an exponential rate in terms of $n$, the number of macro replications. Each macro replication requires running a test of power one, consisting of observing a random walk until the random walk hits a boundary. Let $N_{i}$ denote the number of steps of the $i$ th random walk associated with macro replication $i, i \geq 0$. We complete the macro replication associated with $X_{n}$ by time $T_{n}=\sum_{i=0}^{n} N_{i}, n \geq 0$.

The quantity $T_{n}$ is a better measure of computational complexity than $n$, accounting as it does for the computation in each macro replication. We show in this section that when we measure time using $T_{n}$, which we refer to as "wall-clock time," we should typically expect a convergence rate that is slower than, but arbitrarily close to, that of stochastic approximation.

We first show that we should expect the convergence rate to remain exponential in wall-clock time when $\tilde{g}(x)$ is bounded away from 0 for $x \neq x^{*}$, as in certain edge-detection problems [4] and in certain non-smooth simulation-optimization problems. In the latter case, 14] establishes that under modest conditions a stochastic approximation algorithm can be expected to produce estimates of $x^{*}$ with far larger error that is on the order $O\left(T^{-1}\right)$, where $T$ is the total computational time.

Proposition 1. In addition to the assumptions of Theorem 1, suppose that $\tilde{p}(x)$ is bounded away from $1 / 2$ for $x \neq x^{*}$, i.e., there exists $p_{c}>1 / 2$ such that $\tilde{p}(x) \geq p_{c}$ for all $x \neq x^{*}$. Then there exists $r>0$ such that $e^{r T_{n}}\left|X_{n}-x^{*}\right| \rightarrow 0$ as $n \rightarrow \infty$ a.s.

Proof. Proof. The result follows from the observation that for all $i \geq 0$, conditional on $X_{i}, N_{i} \stackrel{\text { st }}{\leq} N$, where $N$ is a random variable depending only on the lower bound $p_{c}$, with appropriately light tails. Suppose $X_{i}=x<x^{*}$. (The case $X_{i}>x^{*}$ is similar.) Then the random walk $S=\left(S_{k}: k \geq 0\right)$ associated with the power-one test has positive drift $2 \tilde{p}(x)-1 \geq 2 p_{c}-1>0$. Then the stopping time $N_{i}$ of the test (20) is stochastically bounded by the hitting time, $N$, to the (positive only) boundary $\left(k_{m}: m \geq 1\right)$ of the random walk $S$, which has increments equal to 1 with probability $p_{c}$ and -1 with probability $1-p_{c}$.

The random variable $N$ has a finite moment generating function in a neighbourhood of zero. Indeed, for $m \geq 1$,

$$
\begin{aligned}
P(N>m) & \leq P\left(S_{m} \leq k_{m}\right) \\
& =P\left((p-q)-S_{m} / m \geq(p-q)-k_{m} / m\right) \\
& \leq \exp \left[-m\left(p-q-k_{m} / m\right)^{2} / 2\right],
\end{aligned}
$$

where the final inequality uses Hoeffding's inequality, and applies provided that $p-q-k_{m} / m>0$. But $k_{m}$ is of order $(m \ln m)^{1 / 2}$, so for sufficiently large $m, P(N>m)$ decreases exponentially rapidly, and in particular, $N$ has finite mean.

Now, from Theorem 1 there is some $r_{1}>0$ such that $e^{r_{1} n}\left|X_{n}-x^{*}\right| \rightarrow 0$ as $n \rightarrow \infty$ a.s. Thus, it suffices to show that there is some $r>0$ such that $e^{r T_{n}} / e^{r_{1} n} \rightarrow 0$ as $n \rightarrow \infty$ a.s., i.e., that $r T_{n}-r_{1} n \rightarrow-\infty$ as $n \rightarrow \infty$. But this again follows from stochastic domination, since $T_{n}$ is stochastically dominated by the sum of $n+1$ i.i.d. random variables, each of which is distributed according to $N$, and we may then choose $r<r_{1} / E(N)$. 
The situation is more difficult if $\tilde{p}(x)$ approaches $1 / 2$ as $x \rightarrow x^{*}$, which is probably typical. For example, this happens if $g$ is continuous and the error distribution at any $x$ is normal with mean 0 and constant variance. In the case where $\tilde{p}(x)$ approaches $1 / 2$ sufficiently quickly we have the following negative result, based on a result for tests of power one from [5], showing that we should expect the error $X_{n}-x^{*}$ to be stochastically larger than $T_{n}^{-1 / 2}$, which would be the order of the error if $X_{n}$ converged at the canonical rate as typically achieved by stochastic approximation.

Proposition 2. In addition to Assumptions 1-3, suppose that there exists $\epsilon, c>0$ such that $0 \leq$ $\tilde{p}(x)-1 / 2 \leq c\left|x-x^{*}\right|$ for all $x \in\left(x^{*}-\epsilon, x^{*}+\epsilon\right)$. Let $\left(X_{n}: n \geq 0\right)$ be the sequence of medians, where we use a test of power one to determine the updating step. Then $\left(T_{n}^{1 / 2}\left|X_{n}-x^{*}\right|: n \geq 0\right)$ is not tight.

Proof. Proof. It suffices to show that $\left(T_{n}\left(X_{n}-x^{*}\right)^{2}: n \geq 0\right)$ is not tight. Since $T_{n} \geq n$, the result is immediate if $X_{n} \nrightarrow \rightarrow x^{*}$ a.s. as $n \rightarrow \infty$. So suppose $X_{n} \rightarrow x^{*}$ as $n \rightarrow \infty$ a.s. The stated condition on $\tilde{p}(x)$ can be extended to all $x \in[0,1]$ through modification of the constant $c$, and so for all $n$,

$$
\begin{aligned}
T_{n}\left(X_{n}-x^{*}\right)^{2} & \geq N_{n}\left(X_{n}-x^{*}\right)^{2} \\
& \geq N_{n} \frac{\left(\tilde{p}\left(X_{n}\right)-1 / 2\right)^{2}}{c^{2}} .
\end{aligned}
$$

Recall that $N_{n}$ is the time required for the test of power one to conclude. That test is based on a random walk with drift $2 \tilde{p}\left(X_{n}\right)-1$, so the result follows if we show that $\left(N\left(\theta_{n}\right) \theta_{n}^{2}: n \geq 0\right)$ is not tight, where $N(\theta)$ is the stopping time in a power-one test based on a random walk with drift $\theta$, and $\left(\theta_{n}: n \geq 0\right)$ is an arbitrary positive sequence where $\theta_{n} \rightarrow 0$ as $n \rightarrow \infty$.

By passing to a subsequence, we may assume that $\rho_{1} k_{n} / n \leq \theta_{n} \leq \rho_{2} k_{n} / n$, where $k_{n}$ is the value of the upper boundary of the continuation region of the power-one test at time $n$ and $\rho_{1}, \rho_{2} \in(0,1)$ are constants that enable us to employ a result from [5] below. Indeed, if we denote $D_{>}$to be the event that we decide that $\theta>0$ in the power-one test, then from Lemma 4 of [5] we have

$$
\lim \inf _{n \rightarrow \infty} P\left(N\left(\theta_{n}\right) \geq n\right) \geq \lim \inf _{n \rightarrow \infty} P\left(\left\{N\left(\theta_{n}\right) \geq n\right\} \cap D_{>}\right) \geq 1-\gamma,
$$

where $\gamma$ is the confidence parameter of the power-one test as discussed in $₫ 2$. It follows that $P\left(\theta_{n}^{2} N\left(\theta_{n}\right) \geq\right.$ $\left.n \theta_{n}^{2}\right) \geq 1-2 \gamma$ infinitely often. We chose $k_{n}$ to be of order $(2 n \ln n)^{1 / 2}$, so $n \theta_{n}^{2}$ is asymptotically of order $\ln n$, and in particular grows without bound. Thus, for any given $\epsilon<1-2 \gamma$ we will not be able to find a finite $M=M(\epsilon)$ such that $P\left(\theta_{n}^{2} N\left(\theta_{n}\right) \geq M\right)<\epsilon$ for all $n$, which concludes the proof.

While probabilistic bisection cannot achieve the canonical rate that one hopes for with root-finding algorithms, as we see next it can come arbitrarily close. To see how, observe that the runlength $N_{n}$ becomes large when evaluating at a point $X_{n}$ that is "close" to the root. In effect, probabilistic bisection spends a great deal of wall-clock time at points close to the root. This motivates an averaged root estimator of the form

$$
\frac{1}{T_{n-1}} \sum_{i=0}^{n-1} N_{i} X_{i} .
$$

In simulation experiments reported in Chapter 4 of [25] this estimator performs strongly, but we have been unable to establish any theoretical results on its asymptotic convergence behaviour. However, we have been able to establish rates of convergence for a closely related estimator. For $\epsilon \in(0,1 / 2)$, define

$$
\hat{X}_{n}(\epsilon)=\frac{\sum_{i=0}^{n-1} N_{i}^{1 / 2-\epsilon} X_{i}}{\sum_{i=0}^{n-1} N_{i}^{1 / 2-\epsilon}} .
$$

The estimator $\hat{X}_{n}(\epsilon)$ places large weight on points $X_{i}$ at which the runlength $N_{i}$ is large, suggesting close proximity to the root, but the weights are less extreme than those of (12). We next show that this estimator has an error of order at most $T_{n-1}^{-(1 / 2-\epsilon)}$. Since $T_{n-1}$ is a reasonable proxy for the wallclock time needed to compute the estimator, we thus establish a near-canonical convergence rate when $\epsilon$ is small, and since $\epsilon \in(0,1 / 2)$ was arbitrary, we obtain the desired conclusion that the probabilistic bisection algorithm converges at a near-canonical rate.

Theorem 2. Suppose Assumptions 1-3 hold, that we begin with a uniform prior, and that $\tilde{p}(x)-1 / 2 \geq$ $c\left|x-x^{*}\right|$ for some $c>0$. Then $\left\{\left|\hat{X}_{n}(\epsilon)-x^{*}\right| T_{n-1}^{1 / 2-\epsilon}: n \geq 0\right\}$ is tight. 
Remark 1. The assumption that $\tilde{p}$ increases at least linearly near the root is reminiscent of similar conditions, such as a non-zero derivative at the root, for root-finding algorithms. Without such a condition, $\tilde{p}$ could grow so slowly near the root that the runlengths in the tests of power one could be arbitrarily large.

Our proof of Theorem 2 requires a lemma.

Lemma 10. Let $W=\left(W_{n}: n \geq 0\right)$ be a sequence of non-negative random variables, and let $\left(\mathcal{F}_{n}: n \geq 0\right)$ be a filtration. If $\sum_{n=0}^{\infty} E\left(W_{n} \mid \overline{\mathcal{F}}_{n}\right)<\infty$ a.s., then $\sum_{n=0}^{\infty} W_{n}<\infty$ a.s.

Proof. Proof. Suppose if possible that $P(A)>0$ where $A$ is the event $\left\{\sum_{n=0}^{\infty} W_{n}=\infty\right\}$. Since $\sum_{n=0}^{\infty} E\left(W_{n} \mid \mathcal{F}_{n}\right)<\infty$ a.s., there exists $M>0$ such that $P(B(W, \infty)) \geq 1-P(A) / 2$, where we define the events $B(W, m)$ for $m=0,1,2, \ldots, \infty$ by

$$
B(W, m)=\left\{\sum_{n=0}^{m} E\left(W_{n} \mid \mathcal{F}_{n}\right) \leq M\right\} .
$$

Then $P(A \cap B(W, \infty)) \geq P(A)+P(B(W, \infty))-1>0$. If $I_{B}$ is the indicator random variable on the event $B$, then

$$
E\left(\sum_{n=0}^{\infty} W_{n} I_{B(W, \infty)}\right) \geq E\left(\sum_{n=0}^{\infty} W_{n} I_{A \cap B(W, \infty)}\right)=\infty .
$$

On the other hand, by the monotone convergence theorem,

$$
E\left(\sum_{n=0}^{\infty} W_{n} I_{B(W, \infty)}(\cdot)\right)=\lim _{m \rightarrow \infty} E\left(\sum_{n=0}^{m} W_{n} I_{B(W, \infty)}(\cdot)\right) \leq E\left(\sum_{n=0}^{m} W_{n} I_{B(W, m)}\right),
$$

where the last inequality follows since $B(W, \infty) \subseteq B(W, m)$ for any $m<\infty$. In the remainder of the proof, we show that the right-hand side is bounded by $M$, contradicting (13).

We proceed by induction on $m$. The event $B(W, 0)$ is $\mathcal{F}_{0}$ measurable, so $E\left[W_{0} I_{B(W, 0)}\right]=E\left[E\left(W_{0} \mid \mathcal{F}_{0}\right) I_{B(W, 0)}\right] \leq$ $E\left[M I_{B(W, 0)}\right] \leq M$, so the result holds for $m=0$.

Now suppose it holds for (finite) $m-1 \geq 0$. The event $B(W, m)$ is $\mathcal{F}_{m}$ measurable, so that

$$
E\left(\sum_{n=0}^{m} W_{n} I_{B(W, m)}\right)=E\left(\sum_{n=0}^{m} E\left(W_{n} \mid \mathcal{F}_{m}\right) I_{B(W, m)}\right)
$$

implying that we may assume without loss of generality that $W_{m}$ is $\mathcal{F}_{m}$ measurable. Now, define $W^{\prime}=\left(W_{0}^{\prime}, W_{1}^{\prime}, \ldots\right)$ where $W_{n}^{\prime}=W_{n}$ for $n \neq m$ and

$$
W_{m}^{\prime}=\left[M-\sum_{n=0}^{m-1} E\left(W_{n} \mid \mathcal{F}_{n}\right)\right]^{+}
$$

with $[x]^{+}=\max \{x, 0\}$. Then

$$
\begin{aligned}
E\left(\sum_{n=0}^{m} W_{n} I_{B(W, m)}\right) & \leq E\left(\sum_{n=0}^{m} W_{n}^{\prime} I_{B(W, m)}\right) \\
& \leq E\left(\sum_{n=0}^{m} W_{n}^{\prime} I_{B\left(W^{\prime}, m\right)}\right) .
\end{aligned}
$$

Here, (14) follows because $W_{n}^{\prime}=W_{n}$ for all $n=0,1, \ldots, m-1$, and because $W_{m}$ is $\mathcal{F}_{m}$ measurable so that $W_{m}=E\left(W_{m} \mid \mathcal{F}_{m}\right)$, implying that on the event $B(W, m), W_{m}^{\prime} \geq W_{m}$. The inequality (15) follows since $B(W, m) \subseteq B\left(W^{\prime}, m\right)$.

Now define $W^{\prime \prime}=\left(W_{0}^{\prime \prime}, W_{1}^{\prime \prime}, \ldots\right)$ by $W_{n}^{\prime \prime}=W_{n}^{\prime}$ for $n \neq m-1$ and $W_{m-1}^{\prime \prime}=W_{m-1}^{\prime}+W_{m}^{\prime}$. Then

$$
E\left(\sum_{n=0}^{m} W_{n}^{\prime} I_{B\left(W^{\prime}, m\right)}\right)=E\left(\sum_{n=0}^{m-1} W_{n}^{\prime \prime} I_{B\left(W^{\prime \prime}, m-1\right)}\right),
$$

which, by the inductive hypothesis, is bounded by $M$. 
Proof. Proof of Theorem 2 Let $\delta=1 / 2-\epsilon \in(0,1 / 2)$. Then

$$
\begin{aligned}
T_{n-1}^{\delta}\left|\hat{X}_{n}(\epsilon)-x^{*}\right| & =\frac{T_{n-1}^{\delta}}{\sum_{i=0}^{n-1} N_{i}^{\delta}}\left|\sum_{i=0}^{n-1} N_{i}^{\delta}\left(X_{i}-x^{*}\right)\right| \\
& \leq \frac{T_{n-1}^{\delta}}{\left(\sum_{i=0}^{n-1} N_{i}\right)^{\delta}} \sum_{i=0}^{n-1} N_{i}^{\delta}\left|X_{i}-x^{*}\right| \\
& =\sum_{i=0}^{n-1} N_{i}^{\delta}\left|X_{i}-x^{*}\right| \\
& \leq \sum_{i=0}^{\infty} N_{i}^{\delta}\left|X_{i}-x^{*}\right| .
\end{aligned}
$$

We will show, using Lemma 10, that (16) is a finite-valued random variable a.s.,, thereby establishing that $T_{n}^{\delta}\left|X_{n}-x^{*}\right|$ is uniformly dominated (in $n$ ), completing the proof.

First, recall that $N_{i}$ is the sample size used in the power-one test at $X_{i}$, the conditional distribution of which, conditional on $X_{i}$, depends only on the drift $2 \tilde{p}\left(X_{i}\right)-1$. Let $N(\theta)$ generically denote the sample size of the power-one test when the drift is $\theta$. Then [20, 13],

$$
\limsup _{\theta \rightarrow 0} E N(\theta) \theta^{2}\left(\ln \left|\theta^{-1}\right|\right)^{-1}<\infty .
$$

Moreover, a sample-path argument establishes that $E N(\theta)$ is decreasing in $|\theta|>0$, and so it follows that for any $\gamma>0$ there is a $\theta_{0}=\theta_{0}(\gamma)>0$ such that for $\theta \neq 0$,

$$
E N(\theta) \leq c_{1}|\theta|^{-(2+\gamma)} I\left(|\theta| \leq \theta_{0}\right)+c_{2} I\left(|\theta| \geq \theta_{0}\right) .
$$

Here and in what follows, $c_{1}, c_{2}, \cdots$ denote constants that, apart from their existence, do not bear on the argument.

At the point $X_{i}$ with success probability $\tilde{p}\left(X_{i}\right), \theta=\theta\left(X_{i}\right)=2 \tilde{p}\left(X_{i}\right)-1 \geq 2 c\left|X_{i}-x^{*}\right|$ where the inequality is by assumption. Recalling that $X_{i}$ is $\mathcal{G}_{i}$ measurable, it follows from (17) that for $i \geq 0$,

$$
E\left[N_{i} \mid \mathcal{G}_{i}\right] \leq c_{3}\left|X_{i}-x^{*}\right|^{-(2+\gamma)} I\left(\left|X_{i}-x^{*}\right| \leq c_{4}\right)+c_{5} I\left(\left|X_{i}-x^{*}\right|>c_{4}\right) .
$$

Jensen's inequality then allows us to conclude that

$$
E\left[N_{i}^{\delta} \mid \mathcal{G}_{i}\right] \leq c_{3}\left|X_{i}-x^{*}\right|^{-\delta(2+\gamma)} I\left(\left|X_{i}-x^{*}\right| \leq c_{4}\right)+c_{6} I\left(\left|X_{i}-x^{*}\right|>c_{4}\right) .
$$

Let $r>0$ be such that $e^{r n}\left|X_{n}-x^{*}\right| \rightarrow 0$ as $n \rightarrow \infty$ a.s., as in Theorem 1 Next define the index sets $J(1)=\left\{i \geq 0:\left|X_{i}-x^{*}\right|>c_{4}\right\}, J(2)=\left\{i \geq 0: i \notin J(1),\left|X_{i}-x^{*}\right|>e^{-r i}\right\}$ and $J(3)=\{i \geq 0: i \notin$ $J(1) \cup J(2)\}$.

Choose $\gamma>0$ so that $(1-\delta(2+\gamma))>0$. Using (18), we obtain

$$
\begin{aligned}
E\left[N_{i}^{\delta}\left|X_{i}-x^{*}\right| \mid \mathcal{G}_{i}\right] \leq I(i \in & J(1)) c_{6}\left|X_{i}-x^{*}\right|+I(i \in J(2)) c_{3}\left|X_{i}-x^{*}\right|^{1-\delta(2+\gamma)} \\
& \quad+I(i \in J(3)) c_{3} e^{-r(1-\delta(2+\gamma)) i} \\
\leq & I(i \in J(1)) c_{6}+I(i \in J(2)) c_{3}+I(i \in J(3)) c_{3} e^{-r(1-\delta(2+\gamma)) i},
\end{aligned}
$$

since $\left|X_{i}-x^{*}\right| \leq 1$. Summing over $i \geq 0$ and noting that $r(1-\delta(2+\gamma))>0$, we see that

$$
\sum_{i=0}^{\infty} E\left[N_{i}^{\delta}\left|X_{i}-x^{*}\right| \mid \mathcal{G}_{i}\right] \leq c_{6}|J(1)|+c_{3}|J(2)|+c_{7} .
$$

Theorem 1 shows that $J(1)$ and $J(2)$ are finite sets a.s., and the result then follows from (19), (16) and Lemma 10

\section{Additional Proofs}

Proof. A Power One Test Here we establish that (3) has the property that when the drift of the simple random walk $\left(S_{m}: m \geq 0\right)$ with $S_{0}=0$ is $\theta=0$, then the stopping time is finite with probability at most $\gamma$. To that end, Robbins [18] showed via a likelihood ratio test he attributes to Ville [24] that if $B_{m}=$ 
$\sum_{i=1^{m}} Z_{i}$, with $\left(Z_{i}: i \geq 1\right)$ being iid Bernoulli random variables with $P\left(Z_{i}=1\right)=p=1-P\left(Z_{i}=0\right)$, then $P\left(N^{\prime}(p)<\infty\right) \leq \gamma$, where

$$
N^{\prime}(p)=\inf \left\{m \geq 1:\left(\begin{array}{c}
m \\
B_{m}
\end{array}\right) p^{B_{m}}(1-p)^{m-B_{m}} \leq \gamma /(m+1)\right\} .
$$

The "stopping" region for a given $m$ and Bernoulli increments is thus the set of values $k$ such that $P\left(B_{m}=k\right)$ is less than $\gamma /(m+1)$. This set is difficult to explicitly characterize. But for $k \geq m p$, $P\left(B_{m}=k\right) \leq P\left(B_{m} \geq k\right)$, and we can apply Hoeffding's inequality to obtain an upper bound on the latter. This yields a sufficient condition for $P\left(B_{m}=k\right) \leq \gamma /(m+1)$. Thus we can define a new stopping time $N^{\prime \prime}(p)$ such that $N^{\prime \prime}(p) \geq N^{\prime}(p)$, and then $P\left(N^{\prime}(p)<\infty\right) \leq P\left(N^{\prime \prime}(p)<\infty\right) \leq \gamma /(m+1)$. To that end, Hoeffding's inequality yields, for $k \geq m p, P\left(B_{m} \geq k\right) \leq \exp \left(-2 m(k / m-p)^{2}\right)$ which is bounded above by $\gamma /(m+1)$ provided that $k \geq m p+(m(\ln (m+1)-\ln (\gamma)) / 2)^{1 / 2}$. Similarly, for $k \leq m p$, Hoeffding's inequality applied to the left tail yields $P\left(B_{m}=k\right) \leq P\left(B_{m} \leq k\right) \leq \exp \left(-2 m(k / m-p)^{2}\right)$, which yields a symmetric result. Hence we can take

$$
N^{\prime \prime}(p)=\inf \left\{m \geq 1:\left|B_{m}-m p\right| \geq(m(\ln (m+1)-\ln (\gamma)) / 2)^{1 / 2}\right\} .
$$

We can now translate this particular stopping time to the desired stopping time for the random walk $\left(S_{m}: m \geq 0\right)$ with zero drift $\theta$, because $S_{m}=2 B_{m}-m=2\left(B_{m}-m p\right)$ with $p=1 / 2$. Accordingly, we can take as stopping time

$$
\mathcal{N}(0)=\inf \left\{m \geq 1:\left|S_{m}\right| \geq k_{m}\right\}
$$

where $k_{m}=(2 m(\ln (m+1)-\ln (\gamma)))^{1 / 2}$.

Proof. Proof of Lemma 1] The condition $\mu_{n}\left(\left[0, x^{*}\right]\right) \geq 1 / 2+\delta$ is equivalent to $\mu_{n}\left(\left[x^{*}, 1\right]\right) \leq 1 / 2-\delta$ (since $\mu_{n}\left(\left\{x^{*}\right\}\right)=0$ for all $n$ ), which in turn is equivalent to $Y_{n} \geq-\ln (1 / 2-\delta$ ), where

$$
Y_{n}= \begin{cases}\ln \mu_{n}\left(\left[0, x^{*}\right]\right) & \text { if } \mu_{n}\left(\left[0, x^{*}\right]\right)<1 / 2 \\ -\ln \mu_{n}\left(\left(x^{*}, 1\right]\right) & \text { if } \mu_{n}\left(\left[0, x^{*}\right]\right)>1 / 2 \\ 0 & \text { if } \mu_{n}\left(\left[0, x^{*}\right]\right)=1 / 2\end{cases}
$$

The stochastic process $\left(Y_{n}: n \geq 0\right)$ takes negative values when the median is to the right of $x^{*}$, positive values when the median is to the left of $x^{*}$ and equals 0 when the median equals $x^{*}$. Assuming that with probability $1 X_{n} \neq x^{*} \forall n$, we can ignore the knife-edge case where $Y_{n}=0$.

Consider the case where $Y_{n}<0$. Then on the next step, conditional on $X_{n}$ and $Y_{n}$, the mass in $\left[0, x^{*}\right]$ is multiplied by $2 p$ with probability $p\left(X_{n}\right)$ and by $2 q$ with probability $q\left(X_{n}\right)=1-p\left(X_{n}\right)$. Let $\bar{p}$ and $\bar{q}$ be shorthand for $p\left(X_{n}\right)$ and $q\left(X_{n}\right)$ respectively. Hence, if $2 p \mu_{n}\left[0, x^{*}\right]<1 / 2$, i.e., if $\ln (2 p)+Y_{n} \leq \ln (1 / 2)$ then $X_{n}$ will remain in $\left[x^{*}, 1\right]$ on the next step and so, conditional on $X_{n}$ and $Y_{n}$,

$$
Y_{n+1}-Y_{n}= \begin{cases}\ln (2 p) & \text { w.p. } \bar{p}, \\ \ln (2 q) & \text { w.p. } \bar{q} .\end{cases}
$$

However, if $Y_{n}<0$ but $\ln (2 p)+Y_{n} \geq \ln (1 / 2)$, then $X_{n}$ will not necessarily remain in $\left[x^{*}, 1\right]$ on the next step. In this case, $Y_{n}$ can change sign, so that conditional on $X_{n}$ and $Y_{n}$,

$$
Y_{n+1}-Y_{n}= \begin{cases}-\ln \left(1-2 p e^{Y_{n}}\right)-Y_{n} & \text { w.p. } \bar{p} \\ \ln (2 q) & \text { w.p. } \bar{q} .\end{cases}
$$

Similarly, the increment distribution for the case when $Y_{n}>0$ conditional on $X_{n}$ and $Y_{n}$ is, for $Y_{n}-\ln (2 p) \geq-\ln (1 / 2)$,

$$
Y_{n+1}-Y_{n}= \begin{cases}-\ln (2 p) & \text { w.p. } \bar{p} \\ -\ln (2 q) & \text { w.p. } \bar{q}\end{cases}
$$

and if $Y_{n}-\ln (2 p)<-\ln (1 / 2)$ is

$$
Y_{n+1}-Y_{n}= \begin{cases}\ln \left(1-2 p e^{Y_{n}}\right)-Y_{n} & \text { w.p. } \bar{p}, \\ -\ln (2 q) & \text { w.p. } \bar{q} .\end{cases}
$$

We will construct a new stochastic process $\left(\tilde{Y}_{n}: n \geq 0\right)$ such that $\tilde{Y}_{n} \leq Y_{n}$ for all $n$, and such that $\tilde{N}(n)=\left|\left\{k=0, \ldots, n-1: \tilde{Y}_{n} \geq-\ln (1 / 2-\delta)\right\}\right|$ satisfies

$$
P(\tilde{N}(n) \leq \alpha n) \leq \varphi\left(\mu_{0}(A \cup B)\right) e^{-r_{1} n}
$$


for all $n$. This will complete the proof, because $N(n) \geq \tilde{N}(n)$ by pathwise domination, and then $P(N(n) \leq \alpha n) \leq P(\tilde{N}(n) \leq \alpha n)$. We construct this process in two steps.

In the first step we replace $\bar{p}$ with $p$ and $\bar{q}$ with $q$ in both (21) and (22). Next, replace $\bar{p}$ with 1 and $\bar{q}$ with 0 in both (23) and (24). Finally, replace the increment $-\ln (2 p)$ in (23) (which has probability 1) with $-\left(Y_{n}+\ln (1 / 2)\right)$. This last change ensures that if the process starts above level $-\ln (1 / 2)+\ln (2 p)$, it will immediately transition down to $-\ln (1 / 2)$.

After implementing these changes, the new process is a Markov chain on $(-\infty, \infty)$ that is pathwise dominated by $Y$, provided that the new process is initiated at time 0 below $Y_{0}$. The chain also has positive drift on the interval $(-\infty,-\ln (1 / 2)-\ln (2 p))$. This chain is difficult to analyze, however, because it is not $\phi$-irreducible. Accordingly, in the second step we modify the initial value of the chain along with all of the values of the increments by rounding them down to nearby rational values, so as to ensure that the final chain, $\tilde{Y}=\left(\tilde{Y}_{n}: n \geq 0\right)$ lives on the rationals. To this end, let $m$ be a large positive integer that we will specify shortly, and set $\tilde{Y}_{0}=\left\lfloor m Y_{0}\right\rfloor / m$. Then $\tilde{Y}_{0} \leq Y_{0}$ and is rational. Furthermore, replace all of the values in the increment distributions with similar values. For example, in (23) we replace $\ln (2 p)$ with $\lfloor m \ln (2 p)\rfloor / m$ and alter $\ln (2 q)$ similarly. Once this is done for all four increment distributions we obtain a process $\tilde{Y}$ that is stochastically dominated by $Y$, i.e., $\tilde{Y}_{k} \leq Y_{k}$ for all $k \geq 0$. If we now choose $m$ large enough, then $\tilde{Y}$ has positive drift on the interval $(-\infty, \ln (1 / 2)-\ln (2 p))$.

At this point, we have obtained a Markov chain $\tilde{Y}$ that lives on a subset of the rationals and is dominated by $Y$. Moreover it is $V$-uniformly ergodic, where $V(x)=e^{|x| / 2}$ for $x<0, V(x)=1$ for $0 \leq x<-\ln (1 / 2)+\ln (2 p)$ and $V(x)=\sqrt{2}\left(p^{1 / 2}+q^{1 / 2}\right)^{-1}$ for $x \geq-\ln (1 / 2)+\ln (2 p)$. To see why, note that if $Y_{0}=y<\ln (1 / 2)-\ln (2 p)$, then

$$
\begin{aligned}
E V\left(Y_{1}\right) & =p e^{(|y|-\ln (2 p)) / 2}+q e^{(|y|-\ln (2 q)) / 2} \\
& =V\left(Y_{0}\right)\left(p(2 p)^{-1 / 2}+q(2 q)^{-1 / 2}\right) \\
& =V\left(Y_{0}\right)(\sqrt{p / 2}+\sqrt{q / 2})
\end{aligned}
$$

The factor $\zeta=\sqrt{p / 2}+\sqrt{q / 2}<1$ since $\zeta^{2}=1 / 2+\sqrt{p q}$ and $p q<1 / 4$. Also, if $Y_{0}=y>-\ln (1 / 2)+\ln (2 p)$ then $E V\left(Y_{1}\right)=1=\zeta V\left(Y_{0}\right)$.

Let $\pi$ be the stationary distribution of the chain $\tilde{Y}$. From 9 , Theorem 6.3], we conclude that for any subset $D$ of the real line with $\int_{D} \pi(d x) \in(0,1)$ and any $\epsilon>0$,

$$
P\left(\left|\frac{1}{n} \sum_{k=0}^{n-1} \mathbb{I}\left(\tilde{Y}_{k} \in D\right)-\pi(D)\right|>\epsilon\right) \leq \tilde{\varphi}\left(\tilde{Y}_{0}\right) e^{-r_{1} n},
$$

for certain constants $\tilde{\varphi}\left(\tilde{Y}_{0}\right)>0$ and $r_{1}>0$. If we now take $D=[-\ln (1 / 2-\delta), \infty)$ for sufficiently small and positive $\delta$, we find that $\pi(D) \in(0,1)$ and thus

$$
P(|N(n)-n \pi(D)|>\epsilon n) \leq \tilde{\varphi}\left(\tilde{Y}_{0}\right) e^{-r_{1} n} .
$$

Taking $\epsilon=\pi(D) / 2$ and defining $\alpha=\pi(D) / 2$ we obtain the desired result, except for the conclusion about the function $\varphi$. Now, from [9, Theorem 4.1], the quantity $\tilde{\varphi}\left(\tilde{Y}_{0}\right)$ is bounded by $c_{1} V\left(\tilde{Y}_{0}\right)$, which is in turn bounded by $c_{2} V\left(Y_{0}\right)$, since $Y_{0}-\tilde{Y}_{0} \in[0,1 / m)$. The constants $c_{1}$ and $c_{2}$ do not depend on $a$. So if $w=\mu_{0}(A \cup B) \geq 1 / 2$ then $Y_{0}=-\ln \left(\mu_{0}(C)\right)$ and thus $\tilde{Y}_{0} \geq 0$ so that $\tilde{\varphi}\left(\tilde{Y}_{0}\right) \leq \zeta^{-1}$. So for $w \geq 1 / 2$, we can take $\varphi(w)=c_{1} c_{2} \zeta^{-1}$. If $w=\mu_{0}(A \cup B)<1 / 2$ then $Y_{0}=\ln (w)$ and thus $\tilde{Y}_{0} \in[w-1 / m, w)$. It follows that we can take $\varphi(w)=c_{1} c_{2} V(w-1 / m)=c_{1} c_{2} \frac{e^{1 /(2 m)}}{w^{1 / 2}}$. In summary,

$$
\varphi(w)= \begin{cases}c_{1} c_{2} \zeta^{-1} & w \geq 1 / 2 \\ c_{3} w^{-1 / 2} & w<1 / 2\end{cases}
$$

for constants $c_{1}, c_{2}, c_{3}$ that do not depend on $a$.

Proof. Proof of Lemma 2, Measurability is immediate and integrability follows since $\nu_{n}(B)$ can decrease by a factor of at most $q / p$ at each step ( since $\mu_{n}(B)$ decreases by at most a factor $2 q$ and $\mu_{n}(A \cup B)$ increases by at most a factor $2 p$ ).

For the supermartingale property itself, when $n \geq \tau M_{n}=M_{n+1}$ so there is nothing to show. So assume for the remainder of the proof that $n<\tau$. If $X_{n} \in C$ then $M_{n}=M_{n+1}$ and the supermartingale property holds. It remains to check the cases $X_{n} \in A$ and $X_{n} \in B$. 
Suppose that $X_{n} \in A$. Define $a_{n}=\mu_{n}\left(\left[0, X_{n}\right]\right)=1 / 2, b_{n}=\mu_{n}\left(\left(X_{n}, a\right]\right)$, and $c_{n}=\mu_{n}(B)$. If $Z_{n}=1$, i.e., the test concludes that the root lies to the right of $X_{n}$, then

$$
\nu_{n+1}(B)=\frac{2 p c_{n}}{2 q a_{n}+2 p\left(b_{n}+c_{n}\right)}=\frac{2 p c_{n}}{2 p\left(a_{n}+b_{n}+c_{n}\right)-2(p-q) a_{n}}=\frac{\nu_{n}(B)}{1-\frac{p-q}{p} \tilde{a}_{n}},
$$

where $\tilde{a}_{n}=a_{n} /\left(a_{n}+b_{n}+c_{n}\right)=0.5 / \mu_{n}(A \cup B) \in(1 / 2,1)$, and the last step follows by dividing both numerator and denominator by $2 p \mu_{n}(A \cup B)$. Similarly, if $Z_{n}=-1$, then

$$
\nu_{n+1}(B)=\frac{\nu_{n}(B)}{1+\frac{p-q}{q} \tilde{a}_{n}}
$$

Setting $\bar{p}=p\left(X_{n}\right)$ and $\bar{q}=q\left(X_{n}\right)$, we obtain

$$
\begin{aligned}
E\left[\frac{\nu_{n}(B)}{\nu_{n+1}(B)} \mid \mathcal{G}_{n}\right] & =\bar{p}\left(1-(1-q / p) \tilde{a}_{n}\right)+\bar{q}\left(1+(p / q-1) \tilde{a}_{n}\right) \\
& =1+\tilde{a}_{n}(1-q / p)(-\bar{p}+\bar{q} p / q)
\end{aligned}
$$

Now, $-\bar{p}+\bar{q} p / q \leq-p_{c}+q_{c}(p / q)<0$ and thus [26] is bounded above by

$$
1+1 / 2(1-q / p)\left(-p_{c}+q_{c} p / q\right)<1 .
$$

So if $r_{2} \leq-\ln \left[1+1 / 2(1-q / p)\left(-p_{c}+q_{c} p / q\right)\right]$ then the supermartingale property holds when $X_{n} \in A$.

Suppose that $X_{n} \in B$. Redefine $a_{n}=\mu_{n}(A), c_{n}=\mu_{n}\left(\left(a, X_{n}\right]\right)$ and $d_{n}=\mu_{n}\left(\left(X_{n}, x^{*}\right]\right)$. If $Z_{n}=1$, then, similar to the case where $X_{n} \in A$,

$$
\nu_{n+1}(B)=\frac{2 q c_{n}+2 p d_{n}}{2 q\left(a_{n}+c_{n}\right)+2 p d_{n}}=\frac{q\left(c_{n}+d_{n}\right)+(p-q) d_{n}}{q\left(a_{n}+c_{n}+d_{n}\right)+(p-q) d_{n}}=\frac{\nu_{n}(B)+(p / q-1) \tilde{d}_{n}}{1+(p / q-1) \tilde{d}_{n}},
$$

where $\tilde{d}_{n}=d_{n} /\left(a_{n}+c_{n}+d_{n}\right)=\nu_{n}\left(\left(X_{n}, x^{*}\right]\right)$. Also, if $Z_{n}=-1$ then

$$
\nu_{n+1}(B)=\frac{2 p c_{n}+2 q d_{n}}{2 p\left(a_{n}+c_{n}\right)+2 q d_{n}}=\frac{p\left(c_{n}+d_{n}\right)-(p-q) d_{n}}{p\left(a_{n}+c_{n}+d_{n}\right)-(p-q) d_{n}}=\frac{\nu_{n}(B)-(1-q / p) \tilde{d}_{n}}{1-(1-q / p) \tilde{d}_{n}} .
$$

For the sake of notational convenience, let $x=\nu_{n}(B)$ and $y=(p / q-1) \tilde{d}_{n}$. Since $\tilde{d}_{n} \leq \nu_{n}(B)$, we have $0 \leq y \leq(p / q-1) x$, and $x \leq 1$. Then

$$
\begin{aligned}
E\left[\frac{\nu_{n}(B)}{\nu_{n+1}(B)} \mid \mathcal{G}_{n}\right] & =\bar{p} \frac{x+x y}{x+y}+\bar{q} \frac{x-\frac{q}{p} x y}{x-\frac{q}{p} y} \\
& =\bar{p}\left(x+\frac{x(1-x)}{x+y}\right)+\bar{q}\left(x+\frac{x(1-x)}{x-\frac{q}{p} y}\right) \\
& =x+x(1-x)\left(\frac{\bar{p}}{x+y}+\frac{\bar{q}}{x-\frac{q}{p} y}\right) .
\end{aligned}
$$

Holding $x$ fixed, (27) is strictly convex in $y$, so the maximum value over $y \in[0,(p / q-1) x]$ is attained at one of the endpoints of the interval. So either $y=0$, in which case the value of (27) is 1 , or $y=(p / q-1) x$ in which case the value of (27) is

$$
x+(1-x)(\bar{p} q / p+\bar{q} p / q) \leq x+(1-x)\left(p_{c} q / p+q_{c} p / q\right),
$$

where the inequality arises because both sides contain convex combinations of the same two values, with the right-hand side placing larger weight on the larger value. We also have that

$$
p_{c} q / p+q_{c} p / q<p q / p+q p / q=1,
$$

for the same reason, and so $\theta<1$ where $\theta$ is the constant on the left-hand side in (28). Hence, when $y=(p / q-1) x,(27)$ is at most $x+\theta(1-x) \leq 1-\Delta+\theta \Delta<1$. Of the two endpoints, the one with $y=0$ yields the higher bound of 1 . So (27) is at most equal to 1 , thereby proving the supermartingale property when $N(n+1)=N(n)$.

When $N(n+1)=N(n)+1$, i.e., $\mu_{n}\left[0, x^{*}\right] \geq 1 / 2+\delta$, then we want to show that the quantity (27) is bounded away from 1 . To this end, $d_{n} \geq \delta / \mu_{n}\left(\left[0, x^{*}\right]\right) \geq \delta$ so that $y \geq(p / q-1) \delta \geq(p / q-1) \delta x$. 
Thus when we maximize (27), we can now do so over the range $(p / q-1) \delta x \leq y \leq(p / q-1) x$. As above, this is a maximization of a strictly convex function over a convex set, so the maximum is attained at an endpoint. The endpoint $y=(p / q-1) x$ yields the bound $1-\Delta+\theta \Delta<1$ as before. The endpoint $y=(p / q-1) \delta x$ gives a value for (27) of

$$
x+(1-x)\left(\frac{\bar{p}}{1+(p / q-1) \delta}+\frac{\bar{q}}{1-(1-q / p) \delta}\right) .
$$

If we consider this quantity as a strictly convex function of $\delta \in[0,1]$, the maximum is strictly attained at one of the endpoints, and hence since $\delta \in(0,1 / 2)$, for some constant $\theta^{\prime} \in(0,1)$ the value of (29) is at most $x+(1-x) \theta^{\prime} \leq 1-\Delta+\theta^{\prime} \Delta<1$. We have thus shown that (27) is bounded away from 1 .

Proof. Proof of Lemma 4 If $n \geq \eta$ then there is nothing to prove, so suppose that $n<\eta$, and in particular, $\nu_{n}(A)<1 / 2$. Then $\mu_{n}(A)<1 / 2$ so $X_{n} \notin A$. If $X_{n} \in C$ then $L_{n+1}=L_{n}$, so we need only consider the case where $X_{n} \in B$. Let $a_{n}=\mu_{n}(A), c_{n}=\mu_{n}\left(\left(x^{*}-a, X_{n}\right]\right)$ and $d_{n}=\mu_{n}\left(\left(X_{n}, x^{*}\right]\right)$.

If $Z_{n}=+1$, then

$$
\nu_{n+1}(A)=\frac{2 q a_{n}}{2 q\left(a_{n}+c_{n}\right)+2 p d_{n}}=\frac{a_{n}}{a_{n}+c_{n}+d_{n}+(p / q-1) d_{n}}=\frac{\nu_{n}(A)}{1+(p / q-1) \tilde{d}_{n}},
$$

where $\tilde{d}_{n}=d_{n} /\left(a_{n}+c_{n}+d_{n}\right)$ is the conditional mass in $\left(X_{n}, x^{*}\right]$. Similarly, if $Z_{n}=-1$ then

$$
\nu_{n+1}(A)=\frac{2 p a_{n}}{2 p\left(a_{n}+c_{n}\right)+2 q d_{n}}=\frac{a_{n}}{a_{n}+c_{n}+d_{n}-(1-q / p) d_{n}}=\frac{\nu_{n}(A)}{1-(1-q / p) \tilde{d}_{n}} .
$$

Hence, setting $\bar{p}=p\left(X_{n}\right)$ and $\bar{q}=q\left(X_{n}\right)$,

$$
E\left[\frac{\nu_{n+1}(A)}{\nu_{n}(A)}\right]=\frac{\bar{p}}{1+(p / q-1) \tilde{d}_{n}}+\frac{\bar{q}}{1-(1-q / p) \tilde{d}_{n}}=f\left((p / q-1) \tilde{d}_{n}\right)
$$

where

$$
f(y)=\frac{\bar{p}}{1+y}+\frac{\bar{q}}{1-(q / p) y} .
$$

Now, $f$ is strictly convex (in $y$ such that the denominators remain positive), so it attains its maximum over any (finite) interval at an endpoint of the interval. Also, $y=(p / q-1) \tilde{d}_{n} \leq(p / q-1) / 2$ since $d_{n}<1 / 2$ and $\tilde{d}_{n}=d_{n} /\left(1 / 2+d_{n}\right)$. Thus, we need only consider $y \in[0,(p / q-1) / 2]$. Now, $f(0)=1$ and

$$
f((p / q-1) / 2)=\frac{\bar{p}}{1 / 2+p / 2 q}+\frac{\bar{q}}{1 / 2+q / 2 p}=2 q \bar{p}+2 p \bar{q} \leq 4 p q<1 .
$$

The second-to-last inequality results from the fact that $q \bar{p}+p \bar{q}$ is a convex combination of $q$ and $p$, the size of which can be increased by increasing the weight on the larger value, i.e., on $p$. Since $f$ is at most 1 at its endpoints, we have established that (30) is at most 1.

This proves the super martingale property if $N(n+1)=N(n)$. If $N(n+1)=N(n)+1$, then $\tilde{d}_{n} \geq \delta$ and hence $y$ is restricted to the interval $[(p / q-1) \delta,(p / q-1) / 2]$. Over this interval $f$ is strictly convex and thus bounded away from 1.

Proof. Proof of Lemma 5. As in the proof of Lemma 8, we find that

$$
E\left(e^{r_{2}\left(N\left(U_{n}\right)-N\left(V_{n}\right)\right)} \mid \mathcal{G}_{V_{n}}\right) \leq 1 / \nu_{V_{n}}(B) .
$$

At time $i=V_{n}-1, \nu_{i}(B)>1 / 2$, since $\nu_{i}(A)<1 / 2$. In a single time step, the conditional mass in any set can be reduced by at most a factor $q / p$, since $\mu_{i+1}(B) \geq 2 q \mu_{i}(B)$ and $\mu_{i+1}(A \cup B) \leq 2 p \mu_{i}(A \cup B)$. Thus

$$
E\left(e^{r_{2}\left(N\left(U_{n}\right)-N\left(V_{n}\right)\right)} \mid \mathcal{G}_{V_{n}}\right) \leq 2 p / q
$$

For notational convenience, we renormalize by taking time 0 to be the time $V_{n}$, and all probabilities and expectations are conditional on $\mathcal{G}_{V_{n}}$. So now

$$
\begin{aligned}
P\left(U_{n}-V_{n}>k\right) & \leq P\left(N\left(U_{n}-V_{n}\right) \geq N(k)\right) \\
& \leq P\left(N\left(U_{n}-V_{n}\right) \geq N(k), N(k) \geq \alpha k\right)+P(N(k)<\alpha k) \\
& \leq P\left(N\left(U_{n}-V_{n}\right) \geq \alpha k\right)+\varphi\left(\mu_{0}(A \cup B)\right) e^{-r_{1} \alpha k} \\
& \leq E\left[e^{r_{2} N\left(U_{n}-V_{n}\right)}\right] e^{-r_{2} \alpha k}+\varphi\left(\mu_{0}(A \cup B)\right) e^{-r_{1} \alpha k} \\
& \leq 2 \frac{p}{q} e^{-r_{2} \alpha k}+\varphi\left(\mu_{0}(A \cup B)\right) e^{-r_{1} \alpha k} .
\end{aligned}
$$


To complete the proof we establish a uniform bound on $\varphi\left(\mu_{0}(A \cup B)\right)$ by showing that $\mu_{0}(A \cup B) \geq q$. Indeed, the conditional distribution immediately prior to time $V_{n}$ was such that the median was in $B$, since the conditional distribution only changes when the median is in $A \cup B$ and the conditional mass in $A$ was less than $1 / 2$. Thus just prior to time $V_{n}$, the total mass in $A \cup B$ was at least $1 / 2$, this being the mass to the left of the median. Since the mass in any set cannot decrease by more than a factor $2 q$, the mass in $A \cup B$ at time $V_{n}$ is at least $q$.

\section{Acknowledgments.}

This research was partially supported by National Science Foundation grant CMMI-1200315.

\section{References}

[1] S. Asmussen and P. W. Glynn. Stochastic Simulation: Algorithms and Analysis, volume 57 of Stochastic Modeling and Applied Probability. Springer, New York, 2007.

[2] M. V. Burnashev and K. S. Zigangirov. An interval estimation problem for controlled observations. Problemy Peredachi Informatsii, 10(3):51-61, 1974.

[3] M.V. Burnashev and K.S. Zigangirov. On One Problem of Observation Control. Problemy Peredachi Informatsii, 11(3):44-52, 1975.

[4] R. M. Castro and R. D. Nowak. Active learning and sampling. In Alfred O. Hero, David A. Castañón, Douglas Cochran, and Keith Kastella, editors, Foundations and Applications of Sensor Management, pages 177-200. Springer, 2008.

[5] R. H. Farrell. Asymptotic behavior of expected sample size in certain one sided tests. Ann. Math. Statist., 35(1):36-72, 1964.

[6] G. Golubev and B. Levit. Sequential Recovery of Analytic Periodic Edges in Binary Image Models. Mathematical Methods of Statistics, 12(1):95-115, 2003.

[7] M. Horstein. Sequential transmission using noiseless feedback. IEEE Trans. Inform. Theory, 9(3):136-143, 1963.

[8] B. Jedynak, P. I. Frazier, and R. Sznitman. Twenty questions with noise: Bayes optimal policies for entropy loss. Journal of Applied Probability, 49(1):114-136, 2012.

[9] I. Kontoyiannis and S. P. Meyn. Spectral theory and limit theorems for geometrically ergodic Markov processes. Annals of Applied Probability, 13(1):304-362, 2003.

[10] H. J. Kushner and G. Yin. Asymptotic properties of distributed and communicating stochastic approximation algorithms. SIAM Journal on Control and Optimization, 25:1266-1290, 1987.

[11] H. J. Kushner and G. Yin. Stochastic approximation algorithms for parallel and distributed processing. Stochastics, 22:219-250, 1987.

[12] H. J. Kushner and G. Yin. Stochastic Approximation and Recursive Algorithms and Applications. Springer, 2003.

[13] T. L. Lai. Power-one tests based on sample sums. Ann. Statist., 5(5):866-880, 1977.

[14] E. Lim. On the convergence rate for stochastic approximation in the nonsmooth setting. Math. Oper. Res., 36(3):527-537, 2011.

[15] Stephen Pallone, Peter I. Frazier, and Shane G. Henderson. Multisection: Parallelized bisection. In A. Tolk, D. Diallo, I. O. Ryzhov, L. Yilmaz, S. Buckley, and J. A. Miller, editors, Proceedings of the 2014 Winter Simulation Conference, page To appear, Piscataway NJ, 2014. IEEE.

[16] R. Pasupathy and S. Kim. The stochastic root-finding problem: overview, solutions, and open questions. ACM Trans. Model. Comput. Simul., 21(3):19, 2011.

[17] A. Pelc. Searching with known error probability. Theoret. Comput. Sci., 63(2):185-202, 1989.

[18] H. Robbins. Statistical methods related to the law of the iterated logarithm. Ann. Math. Statist., 41(5):1397-1409, 1970.

[19] H. Robbins and S. Monro. A stochastic approximation method. Ann. Math. Statist., 22(3):400-407, 1951. 
[20] H. Robbins and D. Siegmund. The expected sample size of some tests of power one. Ann. Statist., $2(3): 415-436,1974$.

[21] Sergio Rodriguez and Michael Ludkovski. Information directed sampling for stochastic root finding. In Proceedings of the 2015 Winter Simulation Conference, pages 3142-3143. IEEE Press, 2015.

[22] Raphael Sznitman, Aurelien Lucchi, Peter I. Frazier, Bruno M. Jedynak, and Pascal Fua. An optimal policy for target localization with application to electron microscopy. In Sanjoy Dasgupta and David McAllester, editors, Proceedings of the 30th International Conference on Machine Learning (ICML), pages 1-9. Omnipress, 2013.

[23] Theodoros Tsiligkaridis, Brian M Sadler, and Alfred O Hero. Collaborative 20 questions for target localization. IEEE Transactions on Information Theory, 60(4):2233-2252, 2014.

[24] J. Ville. Etude critique de la notion de collectif. Monographies des Probabilites, 3:144, 1939.

[25] Rolf Waeber. Probabilistic bisection search for stochastic root-finding. PhD thesis, Cornell University, Ithaca NY, 2013.

[26] Rolf Waeber, Peter I. Frazier, and Shane G. Henderson. Bisection search with noisy responses. SIAM Journal on Control and Optimization, 51(3):2261-2279, 2013. 